\title{
Network-Like Structure of Lignin in Natural Rubber Matrix to Form High Performance Elastomeric Bio-composite
}

\author{
Treethip Phakkeeree*1, Yuko Ikeda*2., , Hiroyuki Yokohama*1, \\ Pranee Phinyocheep*3, Ritsuko Kitano*4, and Atsushi Kato*4 \\ ${ }^{* 1}$ Graduate School of Science and Technology, Kyoto Institute of Technology, Matsugasaki, Sakyo, Kyoto 606-8585, Japan \\ ${ }^{* 2}$ Faculty of Molecular Chemistry and Engineering, Kyoto Institute of Technology, Matsugasaki, Sakyo, Kyoto 606-8585, Japan \\ ${ }^{* 3}$ Department of Chemistry, Faculty of Science, Mahidol University, Rama VI road, Ratchthewee, Bangkok 10400, Thailand \\ ${ }^{* 4}$ NISSAN ARC, LTD., Natsushima-cho 1, Yokosuka, Kanagawa 237-0061, Japan
}

\begin{abstract}
Lignin functions as an effective reinforcing filler for natural rubber (NR), when it was mixed with NR latex by the soft processing method. The lignin was aggregated, but localized around rubber particles in the NR latex to form a network-like structure of lignin in the NR matrix after drying. The observation clearly showed the role of filler networks for the excellent reinforcement of rubber in the case of organic filler similarly to inorganic fillers.
\end{abstract}

(Received 17 March, 2016; Accepted 2 May, 2016)

\section{Introduction}

Natural rubber (NR) is an important indispensable biomass for modern technology [1]. In many NR products, NR has been manufactured as composites. For example, the rubbery composites of NR and carbon black (CB) had been established their presence among industrial soft materials since long before the word "nanotechnology" came onstage. These materials are reasonably expected to be one of the main players in the $21^{\text {st }}$ century, due to the strong demand for automobile and aircraft tires in the transportation age [1,2].

In terms of sustainable development, on the other hand, bio-fillers such as cellulose nanofibers, bio-silica, and lignin have attracted attention because the products are carbon neutral. Among the bio-fillers, the lignin used in this study was paper-pulping waste, and much research is being focused on the effective use of lignin waste [3-6]. As a promising reuse of the lignin waste, trials of the use of lignin wastes as a filler component in rubbery composites have also been carried out extensively [7-13]. However, lignin is still targeted for further exploration of policy tools for green growth, because lignin is the second most available biopolymer next to cellulose. In addition, inadequate information is available in the existing literature that summarizes the lignin-reinforced polymer composites [6]. Therefore, more effective usage of lignin is needed.

This paper reports a useful morphological effect of lignin to significantly reinforce the lignin/NR biocomposite for the first time. The obtained results also provide new insights into the reinforcement mechanism of rubber by filler filling.

\section{Experimental}

\subsection{Materials and preparation of lignin/NR bio- composites}

Lignin used in this study was commercial sodium lignosulfonate (Pearllex NP, Nippon Paper Chemicals, Co., Ltd.). It was a powder and classified as a high molecular weight grade [14]. The content of sulfonate group was $1.8 \mathrm{mmol} / \mathrm{g}$. This lignin was dissolved in 0.1 $\mathrm{M} \mathrm{NaOH}$ aqueous solution. The concentration of the lignin in the solution was $30 \mathrm{w} / \mathrm{v} \%$. In order to prepare a cross-linked film, $100 \mathrm{~mL}$ of high ammonia

\# corresponding author: E-mail : yuko@kit.ac.jp 
NR latex (ammoniacal latex containing $0.7 \% \mathrm{NH}_{4} \mathrm{OH}$ with $60 \%$ dry rubber content (DRC), Thai Rubber Latex Public Co., Ltd.) was mixed with sulfur crosslinking reagents from Luckyfour Co., Ltd. (50\% sulfur dispersion of 1.5 parts per one hundred rubber by weight (phr), 50\% zinc diethyl dithiocarbamate (ZDEC) dispersion of $1.0 \mathrm{phr}$ and $50 \%$ zinc oxide $(\mathrm{ZnO})$ dispersion of $1.8 \mathrm{phr}$ ) at r.t. in advance, and followed by mixing with $20 \mathrm{~mL}$ of the lignin aqueous solution to give a liquid mixture of lignin and NR. In this study, $10 \mathrm{phr}$ lignin was added to the NR latex for the preparation of lignin/NR bio-composite. The evaporation on a glass plate at r.t. for a few days afforded a soft composite film. The film was subjected to cross-linking at $70{ }^{\circ} \mathrm{C}$ for $4 \mathrm{~h}$ and drying at r.t. under a reduced pressure. A non-filled sample (NR-L0-S-soft) was also prepared by the soft processing method. As reference samples, lignin/NR bio-composites were also prepared from solid NR and the lignin powder by milling, i.e., mechanical mixing on a two-roll mill at r.t. Ten phr lignin, 1.0 phr $\mathrm{ZnO}, 2.0 \mathrm{phr}$ stearic acid, 1.0 phr $N$-cyclohexyl-2-benzothiazole sulfonamide (CBS) and $1.5 \mathrm{phr}$ sulfur were mixed with solid NR (RSS no.1) for sulfur cross-linking. Peroxide cross-linked composites were also prepared, where $10 \mathrm{phr}$ lignin and $1.0 \mathrm{phr}$ dicumyl peroxide were mixed with solid NR or isoprene rubber (IR) on the two-roll mill. The rubber compounds were molded into a sheet of $1 \mathrm{~mm}$ thickness by heat pressing at $140{ }^{\circ} \mathrm{C}$ for $12 \mathrm{~min}$ and $155^{\circ} \mathrm{C}$ for $30 \mathrm{~min}$ for sulfur cross-linking and peroxide cross-linking, respectively. Non-filled sulfur crosslinked sample was also prepared by conventional mixing using the same recipe of curing reagents. In the sample codes, "L", "number", "S" and "P" mean lignin, a lignin content in phr, sulfur cross-linking and peroxide cross-linking, respectively.

\subsection{Measurements}

Tensile properties were measured at r.t. using a custom-made tensile tester (ISUT-2201, Aiesu Giken, Co.). Ring-shaped samples, whose outside and inside diameters were 13.7 and $11.7 \mathrm{~mm}$, respectively, were stretched up at a stretching speed of $100 \mathrm{~mm} / \mathrm{min}$. A stretching ratio $(\alpha)$ is defined as " $\alpha=l / l_{0}$ ", where $l$ is a length after deformation, and $l_{0}$ is an initial length, respectively.

Laser scanning confocal microscopy (LSCM) was conducted using a Nikon Laser Scanning Confocal Microscope System A1R with a 20× objective (numerical aperture $=0.75$, CFI Plan Apochromat 20× $/ 0.75$ DIC M, Nikon) at r.t. The laser wavelength was
$488 \mathrm{~nm}$. A transmission electron microscopy (TEM) was then conducted for NR-L10-S-soft using a transmission electron microscope (JEOL TEM-100U). The accelerating voltage was $80 \mathrm{kV}$. Scanning probe microscopy (SPM) observation was performed using a Bruker AXS Nanoscope III a plus D3100 (Bruker Co.) at r.t. The probes were OMCL-AC160TS (Olympus Co.). The surface of sample was prepared by cutting using an ultra-microtome (LEICA, UC6) at $-100^{\circ} \mathrm{C}$.

A simultaneous synchrotron wide-angle X-ray diffraction (WAXD) and tensile measurements $[15,16]$ were conducted at BL-40XU beam line of SPring- 8 in Harima, Japan [17]. The tensile tester (ISUT-2201) was situated on the beam line and WAXD patterns were recorded using a charge-coupled device camera (ORCA II, Hamamatsu Photonics, Co.) during tensile measurement at r.t. The wavelength of the X-ray was $0.0832 \mathrm{~nm}$, and the camera length was $184.7 \mathrm{~mm}$. Intensity of the incident X-ray was attenuated using a rotating slit equipped on the beam line, and the incident beam was exposed on the sample for $70 \mathrm{~ms}$ every 3s. The obtained WAXD images were processed using "POLAR” (Stonybrook Technology \& Applied Research, Inc. [18]).

\section{Results and discussion}

Fig. 1 shows stress-strain curves of the $10 \mathrm{phr}$ lignin filled composites with the non-filled samples. It was found that the bio-composite prepared by the soft processing showed the largest stresses up to the high strain among the samples. It should be noted that only $10 \mathrm{phr}$ filling of lignin significantly enhanced the tensile properties of the bio-composite, NR-L10-S-soft. The stress at $\alpha=8$ of NR-L10-S-soft was high, more than double that of NR-L10-S-mill. When conventional milling was used, the increase in stress by $10 \mathrm{phr}$ filling was slight, similar to that of the $\mathrm{CB}$ system. Even when the peroxide cross-linking was used to prepare the lignin-filled rubbers, good reinforcement was not achieved not only for NR but for also synthetic natural rubber (IR), as shown by NR-L10-Pmill and IR-L10-P-mill, respectively. This means that the soft processing method is useful for the preparation of high performance lignin/rubber composites.

In order to evaluate morphologies of lignin in the rubber matrixes of the composites, the LSCM was conducted, where any special treatments for the samples were unnecessary because lignin shows an 


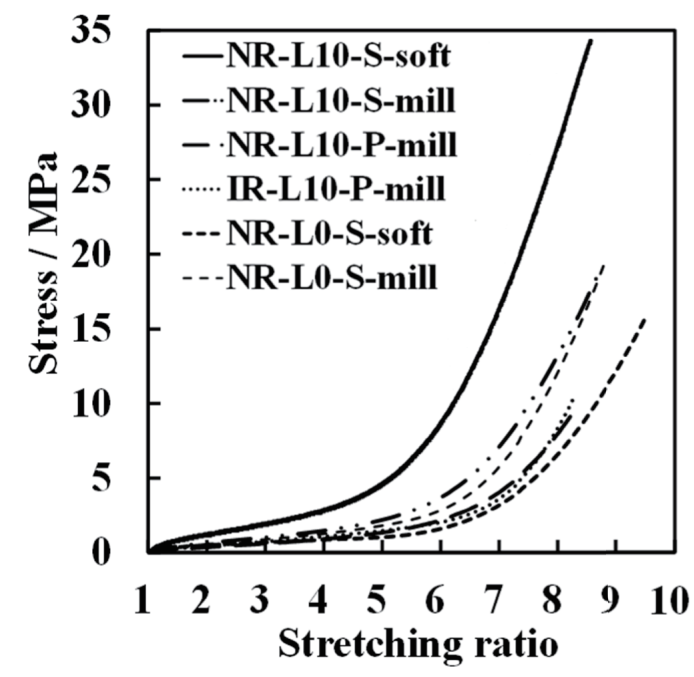

Fig. 1 Tensile stress-strain curves of the composites and non-filled samples.

autofluorescence. Fig. 2 shows LSCM photographs of the composites, lignin powder, dry NR precipitated from the latex using $10 \%$ aqueous acetic acid solution, and the non-filled samples. It is worth noted that NR-L 10-S-soft did not show any lignin powder particles of a similar size to those detected in the lignin milled samples. The observed LSCM image of NR-L10-S-soft was almost same as that of NR-L0-S-soft. Both the dry NR latex and the solid NR show little fluorescence under the objective used in this study; therefore the fluorescence emissions from NR-L10-S-soft and NR-L0S-soft were mainly ascribed to the sulfur cross-linking reagents. These results clearly suggest the presence of a fine dispersion of lignin in NR-L10-S-soft, i.e., a size
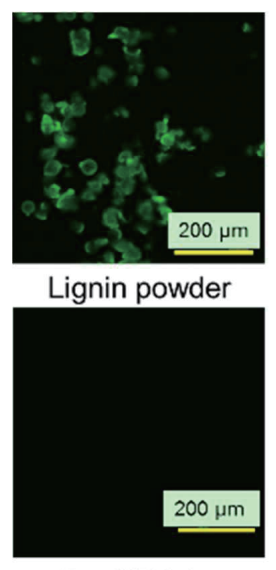

Dry NR latex

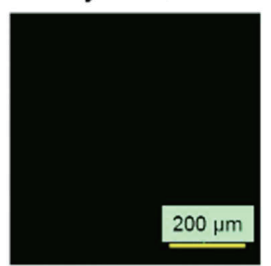

Solid NR

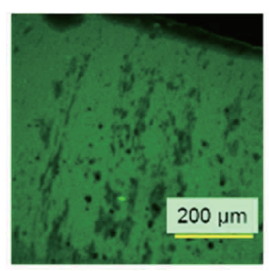

NR-LO-S-soft

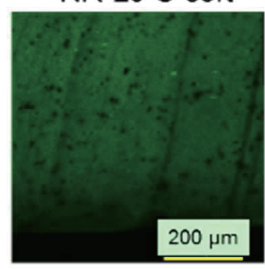

NR-L10-S-soft

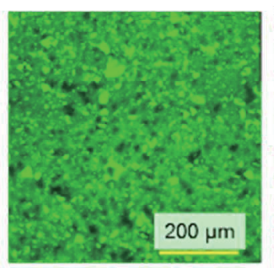

NR-L10-P-mill

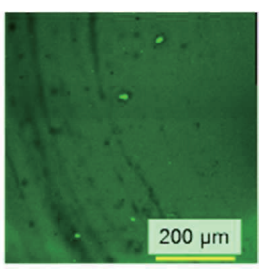

NR-LO-S-mill

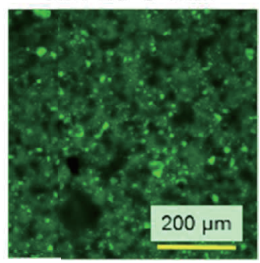

NR-L10-S-mill

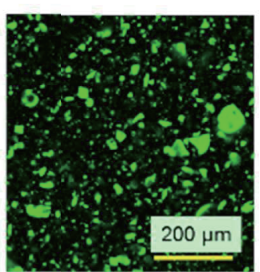

IR-L10-P-mill

Fig. 2 LSCM images of samples. of lignin is indicated to be less than $100 \mathrm{~nm}$ in the NR matrix of NR-L10-S-soft.

TEM observation was then conducted for NR-L 10-S-soft. Fig. 3 shows a TEM photograph, where the two-phase structure in the lignin/NR bio-composite was clearly detected. Dark parts and bright parts represent the lignin and the rubber matrix, respectively. Similarly to the in situ silica filled NR nanocomposites in our previous study [19,20], the lignin was clearly observed to be located around the rubber particles to form a network-like structure of lignin. Inside of rubber matrix, there was little lignin. Namely, the rubber particles in the NR latex provide a template for formation of the lignin network around the NR phases during drying the liquid mixture of lignin and NR. This is similar to the mechanism of the formation of in situ silica network in our previous studies [19-21]. In addition, the network of lignin is considered to partially consist of a continuous phase of the regenerated lignin from the solution. The ionic sites in the sodium lignosulfonate may interact with each other to aggregate like an ionomer after the drying.

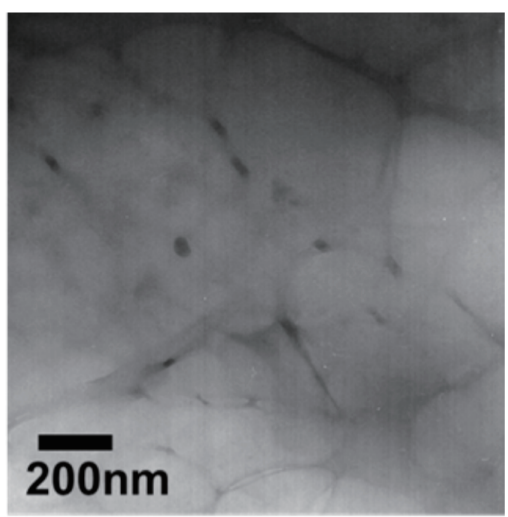

Fig. 3 TEM photograph of NR-L10-S-soft.

The height and phase images of SPM in Fig. 4 (a) and (b), confirm the morphological feature of NR-L10S-soft. The light beige and brown colors show hard and soft phases, i.e., lignin and NR, respectively. This lignin morphology endows NR-L10-S-soft with the excellent tensile properties, as shown in Fig. 1. The results of this study suggest that organic bio-filler of lignin also supports the following idea for the reinforcement of rubber, when the soft processing method was utilized to prepare the nanocomposite: "Not the highest dispersion but to get a certain dispersion that may be favorable to form a networklike structure of nanofiller is to be the best dispersion in terms of rubber reinforcement." Up to now, "the 
better is the dispersion, the more is the reinforcement" has been the popular belief among rubber technologists. However, the observed reinforcement effect in this study clearly shows the role of nanofiller networks for the reinforcement of rubber similarly to the inorganic fillers [21-35].

The effect of "bound rubber" must also be taken into account in the reinforcement of NR-L10-S-soft. Bound rubber is well-known to be rubber that strongly interact with the filler by physical adsorption and chemisorption and/or cross-linking of rubber molecules on the filler surface [36-41], and it has been a central focus in rubber science and technology. The strain-induced crystallization (SIC) behavior of NR-L 10-S-soft may be useful in considering this effect. For example, Fig. 5 shows three-dimensional WAXD patterns at $\alpha=4.5$ of NR-L10-S-soft and NR-L10-S-mill. Crystalline reflections from (200), (120) and (201) are clearly observed for the former, whereas the components of the latter are mainly amorphous. The promotion of SIC was greater for the former than the latter, although the lignin was aggregated around the rubber phases in the former, resulting in a decrease of the interface between the lignin and rubber. The ionic sites in lignin are probably thought to increase the interactions between lignin and NR, as in the case of the hydrophilic sites of in situ silica [19-21,35]. The mechanical strength of lignin alone is not high, but lignin can be used as one component to produce the high-performance rubbery composites if the lignin dispersion is controlled in crystallizable rubbers, as shown in this study. Quantitative discussions on the SIC behaviors will be reported elsewhere.

\section{Conclusions}

Lignin is a waste produced by paper-pulping industries, but it can be used as a good reinforcing

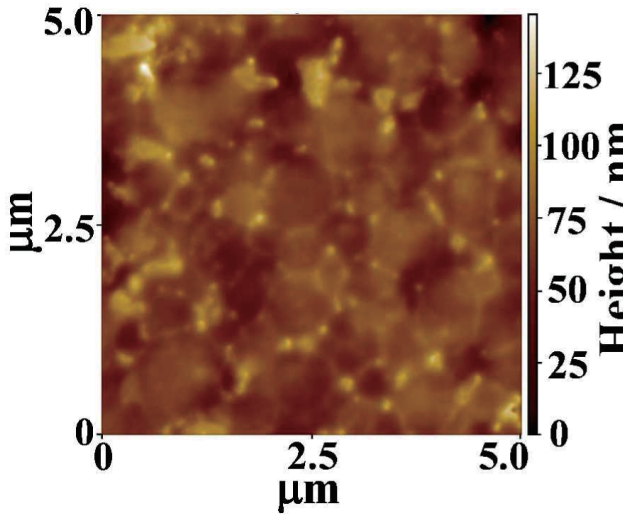

(a)

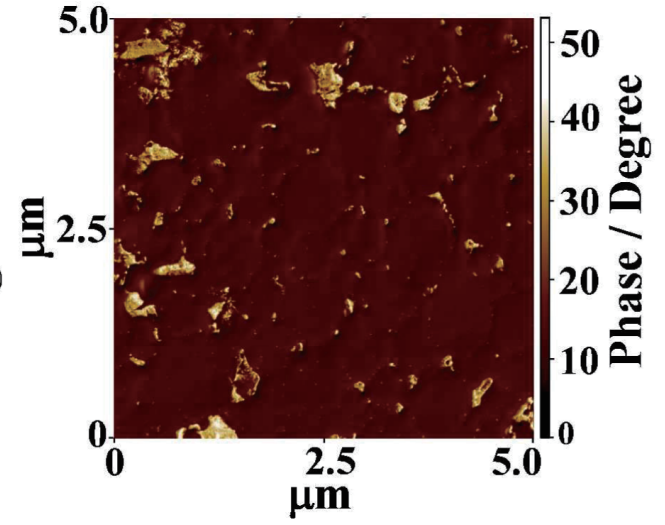

(b)

Fig. 4 SPM images of NR-L10-S-soft. (a) ; a height image and (b) ; a phase image.

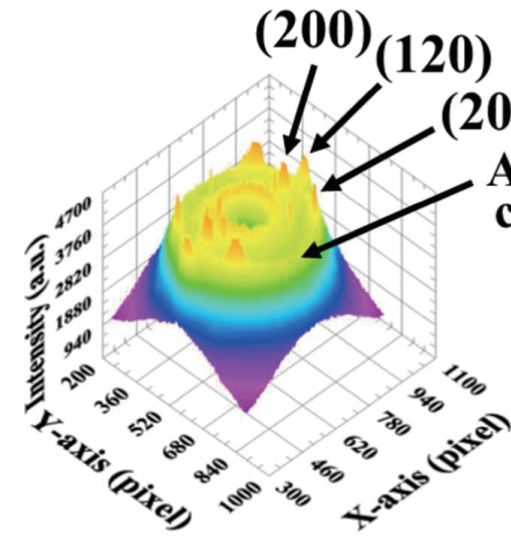

(a)

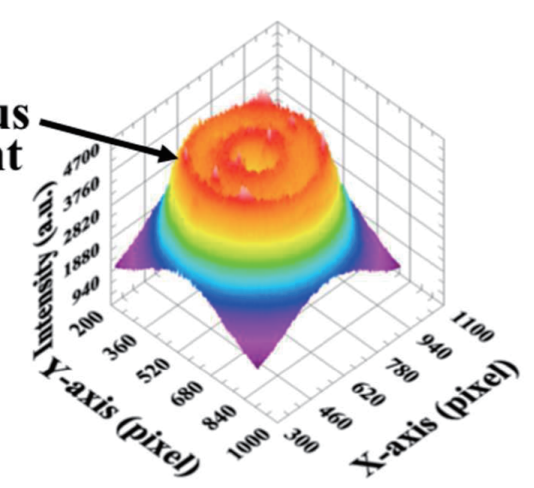

(b)

Fig. 5 Three-dimensional WAXD patterns of NR-L10-S-soft and NR-L10-S-mill at the stretching ratio of 4.5. The stretching direction is parallel to Y-axis. The amorphous scattering and the crystalline reflections from (200), (120) and (201) are indicated by arrows. 
filler for biomass NR when it is mixed with the NR latex using the soft processing. Formation of the network-like agglomerates containing the bound rubber is essential for application of lignin as a high efficient filler for rubber as in the cases of conventional carbon black or particulate silica. Lignin is biodegradable, $\mathrm{CO}_{2}$ neutral, abundantly available in industrial wastes, cheap, and environmentally friendly, with antioxidant, antimicrobial, and stabilizing properties [6]. Therefore, the results of this study will help the development of useful lignin-reinforced polymer composites for sustainable materials.

\section{Acknowledgement}

This work was partially supported by Japan Science and Technology Agency (JST) ALCA program and Kyoto Institute of Technology. The WAXD experiment was performed at the BL40XU in SPring-8 with the approval of the Japan Synchrotron Radiation Research Institute (JASRI) (Proposal No. 2014B1441).

\section{References}

1. "Natural rubber: From the odyssey of the Hevea tree to the age of transportation" (S. Kohjiya Ed.), Smithers RAPRA, Shrewsbury, (2015).

2. Y. Hirata, H. Kondo, Y. Ozawa, "Chemistry, Manufacture and Applications of Natural Rubber" (S. Kohjiya, Y. Ikeda, Eds.), Woodhead/Elsevier, Amsterdam, Ch. 12, pp. 325-352 (2014).

3. “ACS Symposium Series 397” (W. G. Glasser, S. Sarkanen, Eds.), Washington, DC, U.S.A., (1989).

4. T. M. Garver, S. Sarkanen, "Renewable-resource materials : New polymer sources" (C. E. Carraher, Jr., L. H. Sperling, Eds.), Plenum Press, New York, pp. 287-303 (1986).

5. J. H. Lora, W. G. Glasser, J. Polym. Environ., 10, 39 (2002).

6. V. K. Thakur, M. K. Thakur, P. Raghavan, M. R. Kessler, ACS Sustainable Chem. Eng., 2, 1072 (2014).

7. J. J. Keilen, A. Pollak, Ind. Eng. Chem., 39, 480 (1947).

8. J. J. Keilen, W. K. Dougherty, W. R. Cook, Ind. Eng. Chem., 44, 163 (1952).

9. T. R. Griffith, D. W. MacGregor, Ind. Eng. Chem., 45, 380 (1953).

10. F. J. Tibenham, N. S. Grace, Ind. Eng. Chem., 46, 824 (1954).
11. Anon, Chem. Eng. News, 35, 28 (1957).

12. S. Yamashita, S. Kohjiya, "Wood processing and utilization" (J. F. Kennedy, G. O. Philips, P. A. Williams, Eds.), Ellis Horwood, Chichester, Ch. 23 (1989).

13. C. Jiang, H. He, H. Jiang, L. Ma, D. M. Jia, eXPRESS Polymer Letters, 7, 480 (2013).

14. http : //www.npchem.co.jp/english / product/ lignin/

15. Y. Ikeda, Y. Yasuda, S. Makino, S. Yamamoto, M. Tosaka, K. Senoo, S. Kohjiya, Polymer, 48, 1171 (2007).

16. Y. Ikeda, Y. Yasuda, K. Hijikata, M. Tosaka, S. Kohjiya, Macromolecules, 41, 5876 (2008).

17. SPring-8 Web site. http://www.spring8.or.jp/en/

18. S. Toki, I. Sics, S. Ran, L. Liu, B.S. Hsiao, S. Murakami, K. Senoo, S. Kohjiya, Macromolecules, 35, 6578 (2002).

19. A. Tohsan, P. Phinyocheep, S. Kittipoom, W. Pattanasiriwisawa, Y. Ikeda, Polym. Adv. Technol. 23, 1335 (2012).

20. A. Tohsan, Y. Ikeda, "Chemistry, manufacture and applications of natural rubber" (S. Kohjiya, Y. Ikeda, eds.), Woodhead/Elsevier, Cambridge, Ch. 6, pp. 168-192 (2014).

21. A. Tohsan, R. Kishi, Y. Ikeda, Colloid Polym. Sci., 293, 2083 (2015)

22. F. Bueche, "Reinforcement of elastomers" (G. Kraus, Ed,), Interscience, New York, Ch.1, p.1 (1965).

23. “Carbon Black” (J.-B. Donnet, R. C. Bansal, M.-J. Wang, Eds.), Marcel Dekker, New York, (1993).

24. G. Heinrich, M. Klüppel, Adv. Polym. Sci., 160, 1 (2002).

25. M. Klüppel, Adv. Polym. Sci., 164, 1 (2003).

26. S. Kohjiya, A. Katoh, J. Shimanuki, T. Hasegawa, Y. Ikeda, J. Mater. Sci., 40, 2553 (2005).

27. S. Kohjiya, A. Kato, T. Suda, J. Shimanuki, Y. Ikeda, Polymer, 47, 3298 (2006).

28. A. Kato, J. Shimanuki, S. Kohjiya, Y. Ikeda, Rubber Chem. Technol., 79, 653 (2006).

29. S. Kohjiya, A. Kato, Y. Ikeda, Prog. Polym. Sci., 33, 979 (2008).

30. F. Deng, M. Ito, T. Noguchi, Y. A. Kim, M. Endo, Q.-S. Zheng, ACS Nano, 5, 3858 (2011).

31. A. Kato, Y. Ikeda, S. Kohjiya, "Polymer Composites", Vol. 1 (S. Thomas, K. Joseph, S. K. Malhotra, K. Goda, M. S. Sreekala, Eds.), WileyVCH Verlag GmbH \& Co., Weinheim, Ch. 17, pp.515-543 (2012).

32. A. Kato, Y. Ikeda, R. Tsushi, Y. Kokubo, N. Kojima, 
Colloid Polym. Sci., 291, 2101 (2013).

33. A. Kato, Y. Kokubo, R. Tsushi, and Y. Ikeda, "Chemistry, manufacture and applications of natural rubber" (S. Kohjiya, Y. Ikeda, eds.), Woodhead/ Elsevier, Cambridge, Ch. 7, pp. 193-215 (2014).

34. Y. Ikeda, A. Tohsan, Colloid Polym. Sci., 292, 567 (2014).

35. A. Kato, A. Tohsan, S. Kohjiya, T. Phakkeeree, P. Phinyocheep, Y. Ikeda, "Progress in rubber nanocomposites", Woodhead/Elsevier, Amsterdam, Ch. 12, in press.
36. P. B. Stickney, R. D. Falb, Rubber Chem. Technol., 37, 1299 (1964).

37. “Reinforcement of elastomers" (G. Kraus, Ed.), Interscience, New York, (1965).

38. G. Kraus, Rubber Chem. Technol., 38, 1070 (1965).

39. A. K. Sircar, A. Voet, Rubber Chem. Technol., 43, 973 (1970).

40. S. Fujiwara, K. Fujimoto, Rubber Chem. Technol., 44, 1273 (1971).

41. G. Kraus, "Science and Technology of Rubber" (F. R. Eirich, Ed.), Academic Press, New York, Ch. 8, pp. 339-364 (1978). 